\title{
Development of a Population Pharmacokinetic Model for the Diroximel Fumarate Metabolites Monomethyl Fumarate and 2-Hydroxyethyl Succinimide Following Oral Administration of Diroximel Fumarate in Healthy Participants and Patients with Multiple Sclerosis
}

\author{
Mita Kuchimanchi · Howard Bockbrader - Nancy Dolphin • \\ Daniel Epling · Lauren Quinlan · Sunny Chapel · Natasha Penner \\ Received: July 22, 2021 / Accepted: December 16, 2021 / Published online: January 18, 2022 \\ (C) The Author(s) 2022
}

\begin{abstract}
Introduction: Diroximel fumarate (DRF) is a next-generation oral fumarate that is indicated in the USA for relapsing forms of multiple sclerosis (MS). A joint population pharmacokinetic model was developed for the major active metabolite (monomethyl fumarate, MMF) and the major inactive metabolite (2-hydroxyethyl succinimide, HES) of DRF.

Methods: MMF and HES data were included from 341 healthy volunteers and 48 patients with MS across 11 phase I and III studies in which DRF was administered as single or multiple doses. Population modeling was performed with NONMEM version 7.3 with the first-order conditional estimation method.
\end{abstract}

Results: Estimated MMF clearance ( $\left.\mathrm{CL}_{\mathrm{MMF}}\right)$, volume of distribution, and absorption rate constant $(\mathrm{Ka})$ were $13.5 \mathrm{~L} / \mathrm{h}, 30.4 \mathrm{~L}$, and $5.04 \mathrm{~h}^{-1}$, respectively. $\mathrm{CL}_{\mathrm{MMF}}$ and HES

Supplementary Information The online version contains supplementary material available at https:// doi.org/10.1007/s40120-021-00316-6.

M. Kuchimanchi · N. Penner $(\varangle)$

Biogen, Cambridge, MA, USA

e-mail: natalia.penner@gmail.com

H. Bockbrader · N. Dolphin · D. Epling · L. Quinlan

. S. Chapel

Ann Arbor Pharmacometrics Group (A2PG), Ann

Arbor, MI, USA clearance $\left(\mathrm{CL}_{\mathrm{HES}}\right)$ increased with increasing body weight. $\mathrm{CL}_{\mathrm{HES}}$ decreased with decreasing renal function. $\mathrm{CL}_{\mathrm{MMF}}$ and $\mathrm{CL}_{\mathrm{HES}}$ were $28 \%$ and $12 \%$ lower in patients with MS than in healthy volunteers, respectively. Ka was reduced in the presence of low-, medium-, and high-fat meals by $37 \%, 51 \%$, and $67 \%$, respectively, for MMF; and by $34 \%, 49 \%$, and $62 \%$, respectively, for HES.

Conclusions: Age, sex, race, and baseline liver function parameters such as total bilirubin, albumin, and aspartate aminotransferase were not considered to be significant predictors of MMF or HES disposition.

Keywords: 2-Hydroxyethyl succinimide; Diroximel fumarate; Monomethyl fumarate; Multiple sclerosis; Pharmacokinetics; Population pharmacokinetics 


\section{Key Summary Points}

\section{Why carry out this study?}

Diroximel fumarate (DRF) is an approved oral treatment for patients with multiple sclerosis.

DRF is rapidly metabolized in the gastrointestinal tract to produce the major active metabolite monomethyl fumarate (MMF) and the major inactive metabolite 2-hydroxyethyl succinimide (HES).

A joint population pharmacokinetic (PK) model was developed to characterize MMF and HES concentration-time data following oral DRF administration.

\section{What was learned from the study?}

Using our final model, we performed simulations to evaluate the effect of individual covariates on MMF and HES exposures.

Meal fat content, evening dosing, participant body weight, and extent of renal impairment were all determined to impact MMF and HES PK profiles but were not expected to be clinically significant.

\section{INTRODUCTION}

Multiple sclerosis (MS) is an inflammatory demyelinating disease of the central nervous system that results in physical and cognitive disabilities [1]. The chronic and heterogeneous nature of MS results in a wide array of symptoms and the need for lifelong treatment. Diroximel fumarate (DRF) is a novel fumarate approved in the USA for patients with relapsing forms of MS, administered orally as two 231-mg capsules twice daily [2]. Upon oral administration, DRF undergoes rapid esterase cleavage in the gastrointestinal (GI) tract before reaching systemic circulation, producing the major active metabolite monomethyl fumarate (MMF) and the major inactive metabolite 2-hydroxyethyl succinimide (HES) [2, 3]. The minor metabolite RDC-8439 is also produced, resulting in less than $1 \%$ of total DRF-related systemic exposure in humans [3].

MMF is also the active metabolite of dimethyl fumarate (DMF), which is approved in the USA, European Union, and many other regions for relapsing forms of MS [4]. DRF and DMF produce bioequivalent systemic exposure of MMF when administered according to their approved dosing regimens, and therefore are expected to have comparable safety and efficacy profiles [2, 4, 5]. DMF has a well-established benefit-risk profile that has been characterized in more than 537,000 patients, representing more than $1,110,000$ patient-years of exposure [6-9].

GI events are the most common adverse events (AEs) in patients treated with DMF, typically occurring early in treatment, and may lead to treatment disruption or discontinuation in some patients $[6,7,10,11]$. In the phase III head-to-head EVOLVE-MS-2 trial, DRF demonstrated an improved GI tolerability profile compared with DMF in patients with relapsing-remitting MS (RRMS), with fewer days of patient-assessed GI symptoms, lower incidence of GI AEs, and fewer treatment discontinuations due to GI AEs [12]. These improvements are hypothesized to be attributable to the distinct chemical structure of DRF, which may potentially elicit less irritation and reactivity toward off-target receptors in the GI tract [3]. Additionally, interim findings from a phase III study assessing the long-term safety, tolerability, and treatment effect of DRF have demonstrated significant reductions from baseline in annualized relapse rate and gadolinium-enhancing lesion counts, a very low rate (less than 1\%) of treatment discontinuation due to GI AEs, and an overall safety profile consistent with DMF [13].

A comprehensive nonclinical and clinical pharmacology program was conducted to support development of DRF, including eight phase I studies in healthy volunteers, one phase I study in patients with varying degrees of renal failure, and two phase III studies in patients with RRMS. Because DRF is not 
quantifiable in blood plasma following oral administration, pharmacokinetic (PK) analyses conducted to date have been performed with plasma concentrations of MMF and HES. The objectives of this analysis were to develop a population PK model using data collected across 11 DRF clinical studies to characterize the population PK of MMF and HES in healthy volunteers and patients with MS, and to investigate the effects of selected covariates on various PK parameters of MMF and HES to derive a final predictive PK model.

\section{METHODS}

\section{Clinical Studies and Sample Collection}

MMF and HES concentration-time data were collected and pooled across 11 clinical studies of DRF and used to develop the PK model. The following phase I and III studies were included: 001, A102, A103, A104, A105, A106, A109, and A110 (phase I in healthy volunteers); A108 (phase I in healthy volunteers and patients with mild, moderate, or severe renal failure); and EVOLVE-MS-1 and EVOLVE-MS-2 (NCT02634307 and NCT03093324; phase III in patients with RRMS). All clinical studies were conducted in accordance with local and central ethics committees, the Declaration of Helsinki, and the International Conference on Harmonisation Guidelines for Good Clinical Practice. All study participants provided written informed consent.

Following DRF dosing, blood samples were collected according to the PK sampling schedule for each study and used to determine plasma concentrations of MMF and HES. Study designs, participant populations, and PK sampling schedules for all studies included in the analysis are described in Table 1.

\section{Development of the Population PK Model}

The analyses were performed using nonlinear mixed effects modeling methodology as implemented in the computer program NONMEM (version 7.3; ICON plc, Dublin, Ireland). Pre- and postprocessing of data from each modeling step were conducted using SAS (version 9.4), S-PLUS (version 8.2), or $\mathrm{R}$ (version 3.1 .2 or higher) software. Graphical analysis of the data and output from the models was performed using R.

A base model was initially developed to include the following parameters for MMF and HES: absorption rate constant (Ka), clearance $(\mathrm{CL})$, central volume of distribution $(\mathrm{Vc})$, and fractional absorption (F). An assumption was made during the model development that Vc was equal for MMF and HES and thus F for HES was a function of the fractional dose absorbed and dependent on the possible differences in Vc for MMF and HES. Interindividual and interoccasion variability of the PK parameters were incorporated into the base model when applicable using a lognormal random effects model.

Data were classified as outliers using population-weighted residuals (WRES), conditionalweighted residuals (CWRES), and individualweighted residuals (IWRES). Observations with $\mid$ WRES $|>6$,$| CWRES \mid>6$, or $\mid$ IWRES $\mid>6$ were considered potential outliers. The influence of these outliers was evaluated by comparing estimates of the key model parameters (e.g., CL) from model fits on data with and without the outliers. The outliers would be considered influential if key parameter estimates differed by more than $15 \%$. If outliers were influential, subsequent model development was to be performed with and without the outlying observations.

The following covariates were considered for evaluation in the model: participant population (healthy volunteer or patient with MS), sex, dietary intake, dose, and dose time (morning or evening) on Ka and bioavailability; and participant population (healthy volunteer or patient with MS), sex, body weight, age, race, estimated glomerular filtration rate (eGFR), total bilirubin, albumin, and aspartate aminotransferase (AST) on CL and Vc. Selected covariates, based on observed parameter-covariate relationships, were added simultaneously to the base model to produce a full model. A backward elimination procedure with a significance level of $\alpha=0.001$ ( $\Delta$ objective function value less than 10.8 for one degree of freedom) was performed to 
Table 1 Summary of studies included in the population PK analysis

\begin{tabular}{|c|c|c|c|c|c|c|c|}
\hline Study & Phase & $\begin{array}{l}\text { Participant } \\
\text { status }\end{array}$ & Description & DRF dose & $\begin{array}{l}\text { Diet (protocol- } \\
\text { specified) }\end{array}$ & $\begin{array}{l}\text { PK } \\
\text { sample } \\
\text { collection }\end{array}$ & $\begin{array}{l}\text { AM/PM } \\
\text { sampling }\end{array}$ \\
\hline \multirow[t]{2}{*}{001} & 1 & Healthy & $\begin{array}{l}\text { Part 1: single } \\
\text { dose }\end{array}$ & $\begin{array}{l}49,105,210, \\
420,630,840, \\
\text { or } 980 \mathrm{mg}\end{array}$ & $\begin{array}{c}\text { Fasted } 10 \mathrm{~h} \text { before and } \\
4 \mathrm{~h} \text { after study drug }\end{array}$ & $\begin{array}{l}\text { Serial up } \\
\text { to } 24 \mathrm{~h}\end{array}$ & $\mathrm{AM}$ \\
\hline & 1 & Healthy & $\begin{array}{c}\text { Part 2: DRF } \\
\text { vs. DMF } \\
\text { crossover }\end{array}$ & $420 \mathrm{mg}$ & $\begin{array}{c}\text { Fasted } 10 \mathrm{~h} \text { before and } \\
4 \mathrm{~h} \text { after study drug }\end{array}$ & $\begin{array}{l}\text { Serial up } \\
\text { to } 12 \mathrm{~h}\end{array}$ & $\mathrm{AM}$ \\
\hline \multirow[t]{2}{*}{ A102 } & 1 & Healthy & $\begin{array}{l}\text { Part A: single- } \\
\text { dose, } \\
\text { crossover, } \\
\text { food effect }\end{array}$ & $420 \mathrm{mg}$ & $\begin{array}{l}\text { Fasted: } 10 \mathrm{~h} \text { before and } \\
4 \mathrm{~h} \text { after study drug } \\
\text { Fed: fasted } 10 \mathrm{~h} \text { prior to } \\
\text { high-fat/calorie meal } \\
\text { consumed } 30 \mathrm{~min} \\
\text { before study drug }\end{array}$ & $\begin{array}{l}\text { Serial up } \\
\text { to } 12 \mathrm{~h}\end{array}$ & $\mathrm{AM}$ \\
\hline & 1 & Healthy & $\begin{array}{l}\text { Part B: } \\
\text { multiple } \\
\text { dose }\end{array}$ & $\begin{array}{l}\text { 210, 420, or } \\
630 \mathrm{mg} \text { BID } \\
\times 5 \text { days }\end{array}$ & $\begin{array}{l}\text { Days } 1 \text { and } 5 \text { : fasted } 10 \mathrm{~h} \\
\text { before and } 4 \mathrm{~h} \text { after } \\
\text { study drug } \\
\text { Days } 1-5 \text { : fasted } 10 \mathrm{~h} \\
\text { before morning dose }\end{array}$ & $\begin{array}{l}\text { Day 1, } \\
\text { day 5: } \\
\text { serial } \\
\text { up to } \\
10 \mathrm{~h} \\
\text { Days 2, 3, } \\
\text { 4: } 10 \mathrm{~h}\end{array}$ & $\begin{array}{l}\mathrm{AM} \text { and } \\
\mathrm{PM}\end{array}$ \\
\hline A103 & 1 & Healthy & $\begin{array}{l}\text { Single-dose } \\
\text { crossover } \\
\text { DRF vs. } \\
\text { DMF }\end{array}$ & $\begin{array}{l}\text { DRF } 462 \mathrm{mg} \\
\text { DMF } 240 \mathrm{mg}\end{array}$ & $\begin{array}{c}\text { Fasted } 10 \mathrm{~h} \text { before and } \\
4 \mathrm{~h} \text { after study drug }\end{array}$ & $\begin{array}{l}\text { Serial up } \\
\text { to } 72 \mathrm{~h}\end{array}$ & $\mathrm{AM}$ \\
\hline A104 & 1 & Healthy & $\begin{array}{l}\text { Single-dose } \\
\text { crossover } \\
\text { DRF vs. } \\
\text { DMF }\end{array}$ & $\begin{array}{l}\text { DRF } 462 \mathrm{mg} \\
\text { DMF } 240 \mathrm{mg}\end{array}$ & $\begin{array}{l}\text { Fasted } 10 \mathrm{~h} \text { prior to } \\
\text { high-fat/calorie meal } \\
\text { consumed } 30 \mathrm{~min} \\
\text { before study drug }\end{array}$ & $\begin{array}{l}\text { Serial up } \\
\text { to } 48 \mathrm{~h}\end{array}$ & $\mathrm{AM}$ \\
\hline A105 & 1 & Healthy & $\begin{array}{l}\text { Period 1: } \\
\text { unlabeled } \\
\text { DRF single } \\
\text { dose, mass } \\
\text { balance }\end{array}$ & $462 \mathrm{mg}$ & $\begin{array}{l}\text { Fasted } 10 \mathrm{~h} \text { before and } \\
4 \mathrm{~h} \text { after study drug }\end{array}$ & $\begin{array}{l}\text { Serial up } \\
\text { to } \\
120 \mathrm{~h}\end{array}$ & $\mathrm{AM}$ \\
\hline A106 & 1 & Healthy & $\begin{array}{l}\text { 3-way } \\
\text { crossover } \\
\text { study with } \\
5 \% \text { or } 40 \% \\
\text { alcohol }\end{array}$ & $462 \mathrm{mg}$ & Fasted & $\begin{array}{l}\text { Serial up } \\
\text { to } 72 \mathrm{~h}\end{array}$ & $\mathrm{AM}$ \\
\hline
\end{tabular}


Table 1 continued

\begin{tabular}{|c|c|c|c|c|c|c|c|}
\hline$\overline{\text { Study }}$ & Phase & $\begin{array}{l}\text { Participant } \\
\text { status }\end{array}$ & Description & DRF dose & $\begin{array}{l}\text { Diet (protocol- } \\
\text { specified) }\end{array}$ & $\begin{array}{l}\text { PK } \\
\text { sample } \\
\text { collection }\end{array}$ & $\begin{array}{l}\mathrm{AM} / \mathbf{P M} \\
\text { sampling }\end{array}$ \\
\hline A108 & 1 & $\begin{array}{l}\text { Renal } \\
\text { impairment }\end{array}$ & Single dose & $462 \mathrm{mg}$ & $\begin{array}{l}\text { Fasted } 8 \mathrm{~h} \text { before and } 4 \mathrm{~h} \\
\text { after dose } \\
\text { Light snack } 2 \mathrm{~h} \text { pre dose }\end{array}$ & $\begin{array}{l}\text { Serial up } \\
\text { to } \\
216 \mathrm{~h}\end{array}$ & $\mathrm{AM}$ \\
\hline A109 & 1 & Healthy & $\begin{array}{l}\text { Single dose, } \\
\text { 4-way } \\
\text { crossover } \\
\text { food effect }\end{array}$ & $462 \mathrm{mg}$ & $\begin{array}{l}\text { Fasted: } 10 \mathrm{~h} \text { before and } \\
4 \mathrm{~h} \text { after study drug } \\
\text { Fed: low- or medium-fat/ } \\
\text { calorie meal consumed } \\
30 \text { min before drug, no } \\
\text { food } 4 \mathrm{~h} \text { after study } \\
\text { drug }\end{array}$ & $\begin{array}{l}\text { Serial up } \\
\text { to } 48 \mathrm{~h}\end{array}$ & $\mathrm{AM}$ \\
\hline A110 & 1 & Healthy & $\begin{array}{l}\text { Multiple } \\
\text { doses, QTc }\end{array}$ & $\begin{array}{l}462 \mathrm{mg} \text { BID on } \\
\text { days } 2-5 \text {, QD } \\
\text { day } 6 \\
924 \mathrm{mg} \text { BID on } \\
\text { days } 7-10 \text {, } \\
\text { QD day } 11\end{array}$ & $\begin{array}{l}\text { Fasted } 10 \mathrm{~h} \text { before and } \\
4 \mathrm{~h} \text { after drug }\end{array}$ & $\begin{array}{l}\text { Day 6, } \\
\text { day 11: } \\
\text { serial } \\
\text { up to } \\
24 \mathrm{~h}\end{array}$ & $\mathrm{AM}$ \\
\hline $\begin{array}{l}\text { EVOLVE- } \\
\text { MS-1 }\end{array}$ & 3 & $\begin{array}{l}\text { Patients with } \\
\text { MS }\end{array}$ & $\begin{array}{l}\text { Open-label } \\
\text { study up to } \\
96 \text { weeks }\end{array}$ & $\begin{array}{l}\text { De novo } \\
\text { participants: } \\
231 \mathrm{mg} \text { BID } \\
\text { for week } 1 \text {, } \\
\text { then } 462 \mathrm{mg} \\
\text { BID } \\
\text { Rollover } \\
\text { participants: } \\
462 \text { mg BID }\end{array}$ & $\begin{array}{l}\text { Take on an empty } \\
\text { stomach, at least } 1 \mathrm{~h} \\
\text { before or } 2 \mathrm{~h} \text { after } \\
\text { eating or drinking } \\
\text { anything but water }\end{array}$ & $\begin{array}{l}\text { Day } 1 \text {, } \\
\text { day 29: } \\
\text { serial } \\
\text { up to } \\
8 \mathrm{~h} \\
\text { Day } 15, \\
\text { day } 57 \text { : } \\
\text { pre and } \\
2-3 \mathrm{~h} \\
\text { post }\end{array}$ & $\begin{array}{c}\mathrm{AM} \text { or } \\
\mathrm{PM}\end{array}$ \\
\hline
\end{tabular}


Table 1 continued

\begin{tabular}{|c|c|c|c|c|c|c|c|}
\hline Study & Phase & $\begin{array}{l}\text { Participant } \\
\text { status }\end{array}$ & Description & DRF dose & $\begin{array}{l}\text { Diet (protocol- } \\
\text { specified) }\end{array}$ & $\begin{array}{l}\text { PK } \\
\text { sample } \\
\text { collection }\end{array}$ & $\begin{array}{l}\text { AM/PM } \\
\text { sampling }\end{array}$ \\
\hline \multirow[t]{2}{*}{$\begin{array}{c}\text { EVOLVE- } \\
\text { MS-2 }\end{array}$} & 3 & $\begin{array}{l}\text { Patients with } \\
\text { MS }\end{array}$ & $\begin{array}{l}\text { DRF and } \\
\text { DMF }^{\mathrm{a}} \\
\text { Part A } \\
\text { (exploratory) }\end{array}$ & $\begin{array}{l}231 \mathrm{mg} \text { BID } \\
\text { week } 1 \\
462 \mathrm{mg} \text { BID } \\
\text { weeks 2-5 }\end{array}$ & $\begin{array}{l}\text { With or without food, } \\
\text { avoid taking with a } \\
\text { high-fat/calorie meal }\end{array}$ & $\begin{array}{l}\text { Day 1, } \\
\text { day 29: } \\
\text { serial } \\
\text { up to } \\
8 \mathrm{~h}\end{array}$ & $\mathrm{AM}$ \\
\hline & & & & & & $\begin{array}{l}\text { Days } 15 \text {, } \\
\text { day } 36: \\
\text { pre and } \\
2-3 \mathrm{~h} \\
\text { post }\end{array}$ & $\begin{array}{c}\text { AM or } \\
\text { PM }\end{array}$ \\
\hline
\end{tabular}

$A M$ morning dose, $B I D$ twice daily, $D M F$ dimethyl fumarate, $D R F$ diroximel fumarate, $M S$ multiple sclerosis, $P K$ pharmacokinetic, $P M$ evening dose, $Q D$ single dose, $Q T c$ QT interval corrected for heart rate

${ }^{a}$ DMF dosing was $120 \mathrm{mg}$ BID for week 1 and $240 \mathrm{mg}$ BID for weeks 2-5

identify a parsimonious preliminary final model. Standard goodness-of-fit plots were used to assess model fit at each stage of model development. The predictive performance of the final model was evaluated using an internal visual predictive check. Additional information on the overall modeling strategy, including development of the base and final models, standard goodness-of-fit plots, and subsequent covariate analyses, can be found in the Electronic Supplementary Material (ESM).

\section{RESULTS}

\section{Study Population}

There were 389 participants in the overall study population across the 11 clinical studies, including 341 (88\%) healthy volunteers and 48 (12\%) patients with MS. In study participants at baseline, median participant age was 35 years (range $18-75$ years), 51\% were male, and 66\% were white. Median body weight was $78 \mathrm{~kg}$ (range $47.4-126.3 \mathrm{~kg}$ ). The majority of participants $(75.5 \%)$ had normal renal function; $20.0 \%, 2.3 \%$, and $2.0 \%$ had mild $(60-89 \mathrm{~mL} /$ min at screening), moderate $(30-59 \mathrm{~mL} / \mathrm{min}$ at screening), and severe (less than $30 \mathrm{~mL} / \mathrm{min}$ at screening) renal impairment, respectively, based on eGFR. A summary of participant baseline characteristics by study and for the overall study population can be found in Tables S1 and S2 of the ESM.

Participants received DRF in doses ranging from 49 to $980 \mathrm{mg}$, with the majority (69\%; 270/389) receiving DRF $462 \mathrm{mg}$ (approved dose). DRF was administered to healthy participants in the fasted state $(n=252)$ or with lowfat $(n=47)$, medium-fat $(n=47)$, or high-fat $(n=58)$ meals, and to patients with MS $(n=48)$ with or without a meal of unknown fat content. Some phase I study participants received DRF with meals of more than one type of fat content because of crossover study designs.

In the final analysis dataset, there were 4694 MMF and 8088 HES concentration samples available for the population PK analysis (Tables S3 and S4 of the ESM).

\section{Model Development}

A joint metabolite PK model was developed to characterize MMF and HES concentration-time data following DRF administration. Because 
A

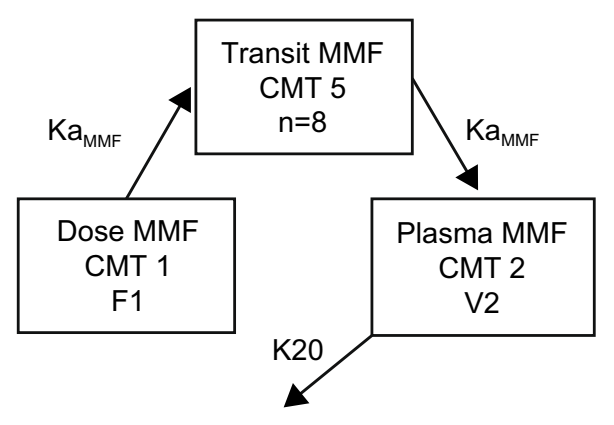

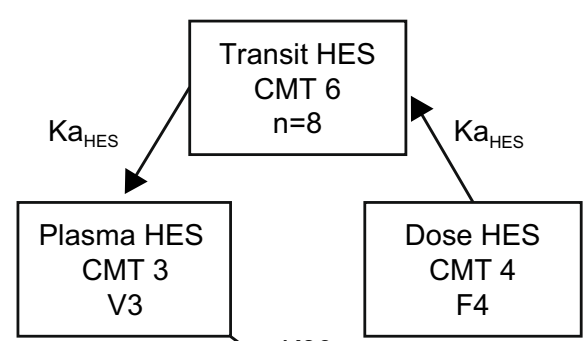

K30

B

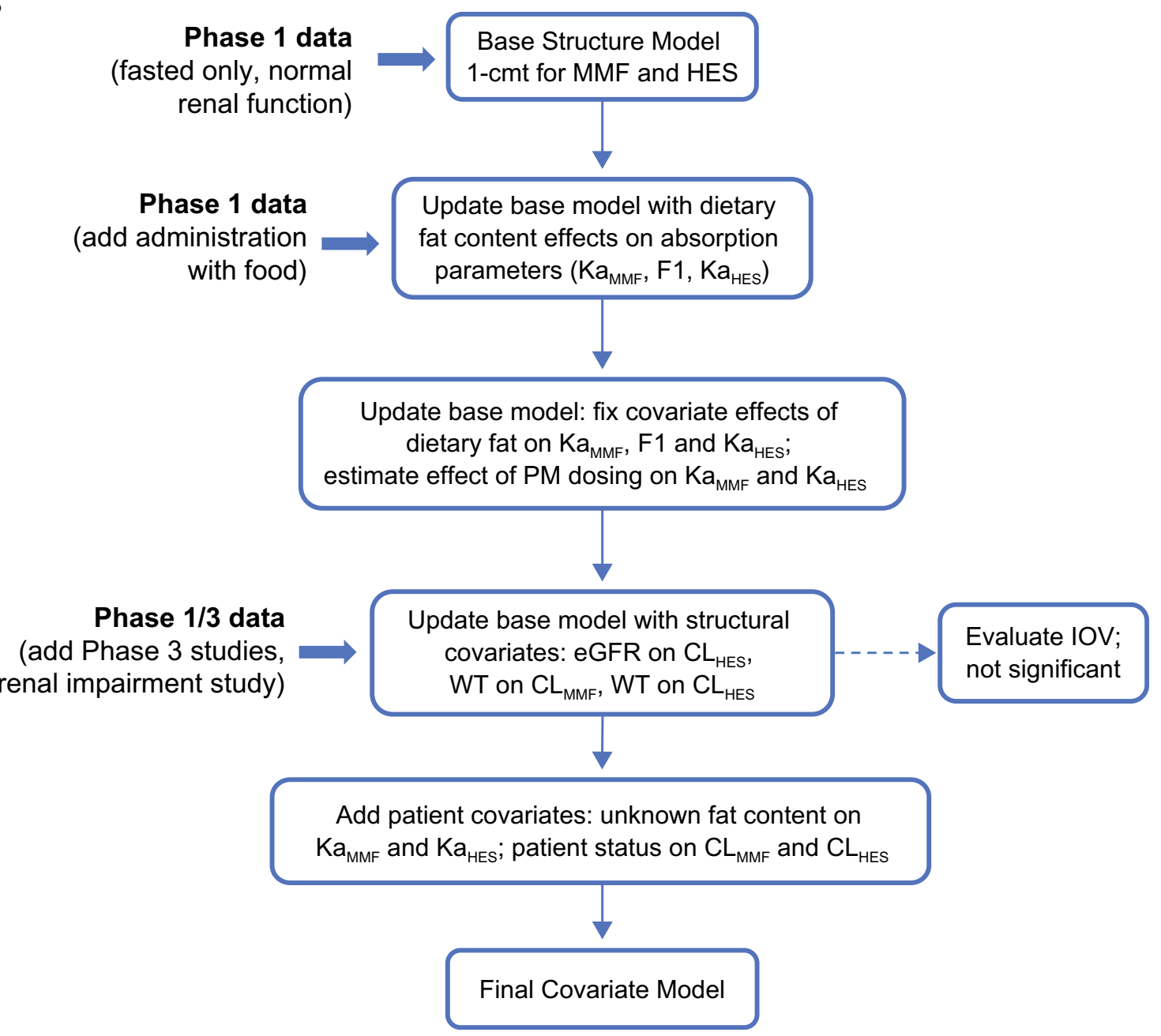

Fig. 1 Pharmacokinetic model (a) and summary of model development steps $(\mathbf{b})$. A single term was estimated for the $\mathrm{Vc}$ in the population PK model (i.e., V2 = V3). $\mathrm{CL}_{\mathrm{HES}}$ clearance of HES, $\mathrm{CL}_{\mathrm{MMF}}$ clearance of MMF, CMT compartment, eGFR estimated glomerular filtration rate, F1 bioavailability of MMF, F4 bioavailability of HES, HES 2-hydroxyethyl succinimide, IOV interoccasion variability, K20 elimination rate constant for $\mathrm{MMF}\left(=\mathrm{CL}_{\mathrm{MMF}} / \mathrm{V} 2\right)$,
K30 elimination rate constant for HES (= $\left.\mathrm{CL}_{\mathrm{HES}} / \mathrm{V} 3\right)$, $\mathrm{Ka}_{\mathrm{HES}}$ absorption rate constant of HES, Ka $\mathrm{a}_{\mathrm{MMF}}$ absorption rate constant of MMF, MMF monomethyl fumarate, PK pharmacokinetics, PM evening dose, V2 central CMT distribution volume of MMF, V3 central CMT distribution volume of HES, Vc central volume of distribution, WT body weight 
DRF is rapidly metabolized and its plasma concentration cannot be measured, DRF dose was inputted to the model as molar equivalents of MMF and HES as follows: dose of MMF or HES $($ molar $)=$ dose of DRF $(\mathrm{g}) /$ molecular weight (DRF). PK data were described using a onecompartment disposition model with transit compartment absorption for MMF and HES as well as first-order elimination for each metabolite (Fig. 1a). The model, including eight transit compartments, was found to adequately describe the delayed absorption of both MMF and HES. Bioavailability was estimated for MMF in the population analysis (a reflection of bioavailability and possible differences in Vc for MMF and HES) and fixed at 0.6 for HES based on results of a clinical mass balance study (Study ALK8700-A105) [2]. Model parameters included CL of MMF (CL $\left.L_{M M F}\right), C L$ of HES (CL $\mathrm{CLES}_{\mathrm{HES}}$ ), and a single $\mathrm{Vc}$ for both metabolites based on the approach by Bertrand et al. [14]. No evidence of dose dependency for MMF or HES PK was demonstrated over the dose range studied.

The model was initially developed using PK data from single-dose studies in healthy participants and later progressed with additional studies and new covariates to improve model fit (Fig. 1b). Factors known to influence the absorption of MMF and HES were incorporated as structural covariates in the base model as follows: meal fat content and evening dosing on $\mathrm{Ka}$; meal fat content on bioavailability (MMF only); baseline body weight on CL and Vc; and eGFR on CL (HES only). In addition, a lag time was estimated to account for delayed absorption of HES with evening dosing. Absorption parameters were estimated using phase I data and then fixed to the estimated values in the development of the final model, which included pooled phase I and III data. Once a base model was established with combined phase I and III data, patient-specific covariates were evaluated.

Covariate-parameter relationships were identified for testing in subsequent covariate analyses. $R^{2}$ values were calculated to assess the correlation between continuous covariates and parameter values, and categorical covariates were assessed by visual inspection. There were no continuous covariates with $R^{2}>0.05$, hence none were evaluated further during covariate analysis. From among the prespecified covariates considered, excluding those identified as structural covariates, the following four covariate-parameter relationships were selected for evaluation using a full model approach: patient status on $\mathrm{Ka}$ of MMF $\left(\mathrm{Ka}_{\mathrm{MMF}}\right)$ and HES $\left(\mathrm{Ka}_{\mathrm{HES}}\right)$, and patient status on $\mathrm{CL}_{\mathrm{MMF}}$ and $\mathrm{CL}_{\mathrm{HES}}$. $\mathrm{A}$ backward elimination procedure was used to demonstrate statistical significance $(\alpha=0.001)$ for all four covariates, which were therefore retained in the final model.

Additional information on development of the base model and PK parameter estimates for the base model can be found in the Supplementary Results and Tables S5 and S6 of the ESM.

\section{Final Model}

Parameter estimates for the final model are shown in Table 2 . Interindividual variability (percentage coefficient of variation) was $37 \%$ for $\mathrm{Ka}_{\mathrm{MMF}}$ and $42 \%$ for $\mathrm{Ka}_{\mathrm{HES}} ; 24 \%$ for $\mathrm{CL}_{\mathrm{MMF}}$ and $18 \%$ for $\mathrm{CL}_{\mathrm{HES}} ;$ and $20 \%$ for the combined metabolite Vc. Separate residual error terms were estimated for MMF and HES under various dosing conditions (fasted, fed, morning/evening administration). In general, residual error estimates were higher for MMF (90-112\%) compared with HES (18-47\%).

Based on rate constants $\left(\mathrm{Ka}_{\mathrm{MMF}}\right.$ and $\left.\mathrm{Ka}_{\mathrm{HES}}\right)$ in the transit absorption model, the mean transit time is estimated as $1.6 \mathrm{~h}$ for MMF and $2.5 \mathrm{~h}$ for HES.

\section{Effects of Covariates}

Using the final model, simulations were performed to assess the effect of individual covariates on MMF and HES exposures. Each specified covariate value (i.e., test condition) was evaluated relative to a reference set of covariates. Exposure parameters included steady state maximum plasma drug concentration over the $0-12-\mathrm{h}$ dosing interval $\left(C_{\max 0-12 \mathrm{~h}, \mathrm{ss}}\right)$ and steady state area under the plasma drug concentration-time curve over the 0-12-h dosing interval $\left(\mathrm{AUC}_{0-12 \mathrm{~h}, \mathrm{ss}}\right)$. Simulations were 


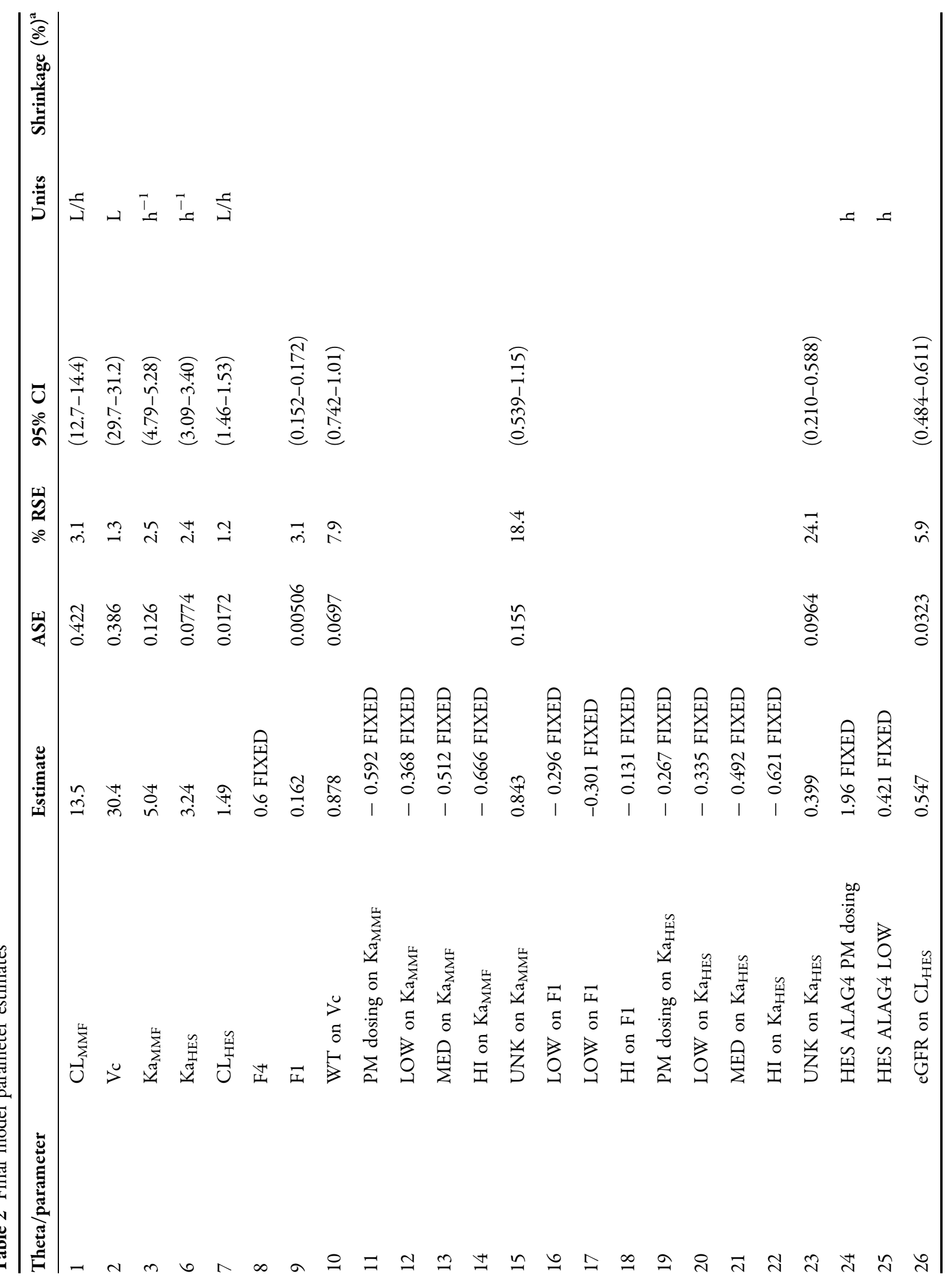




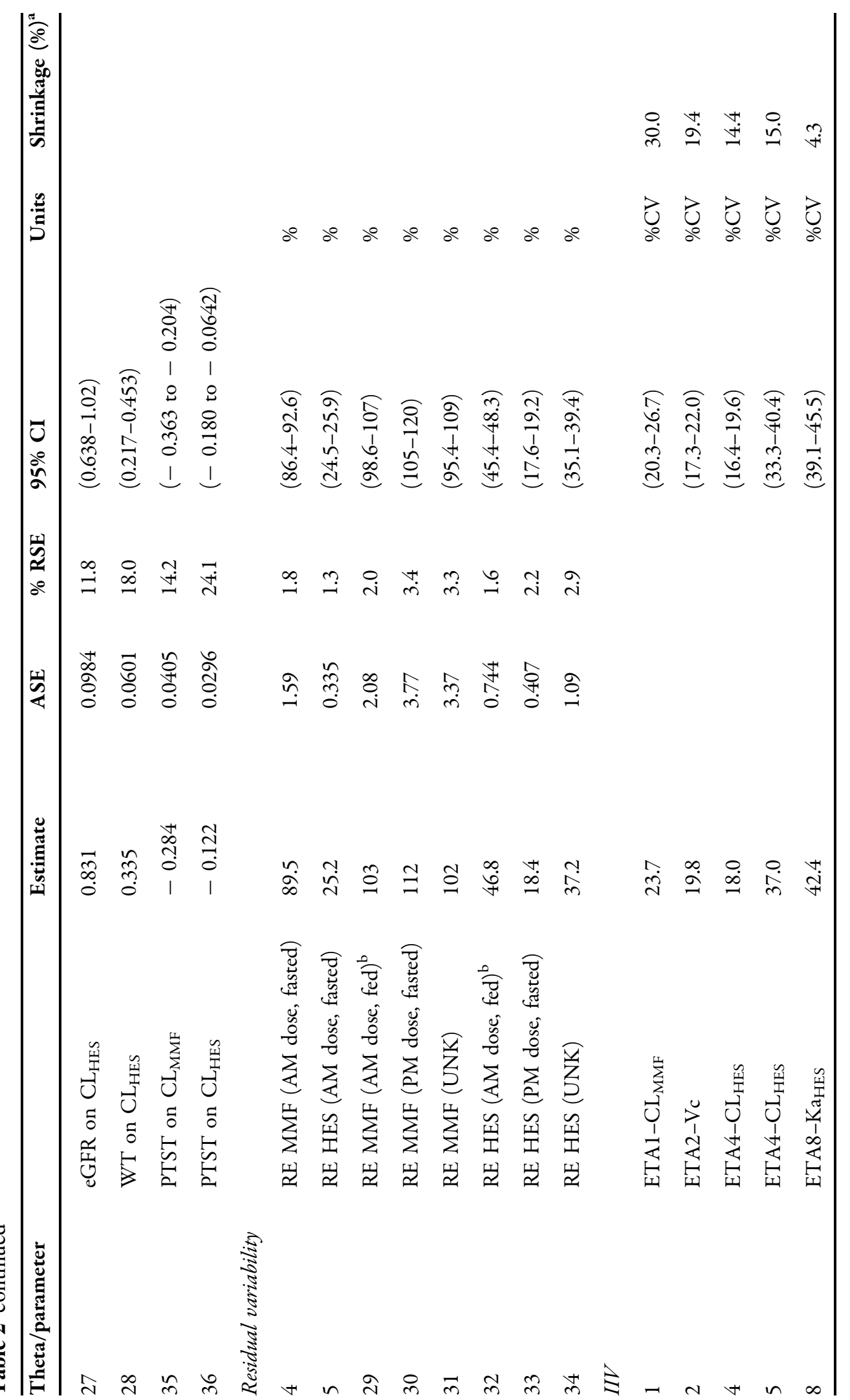




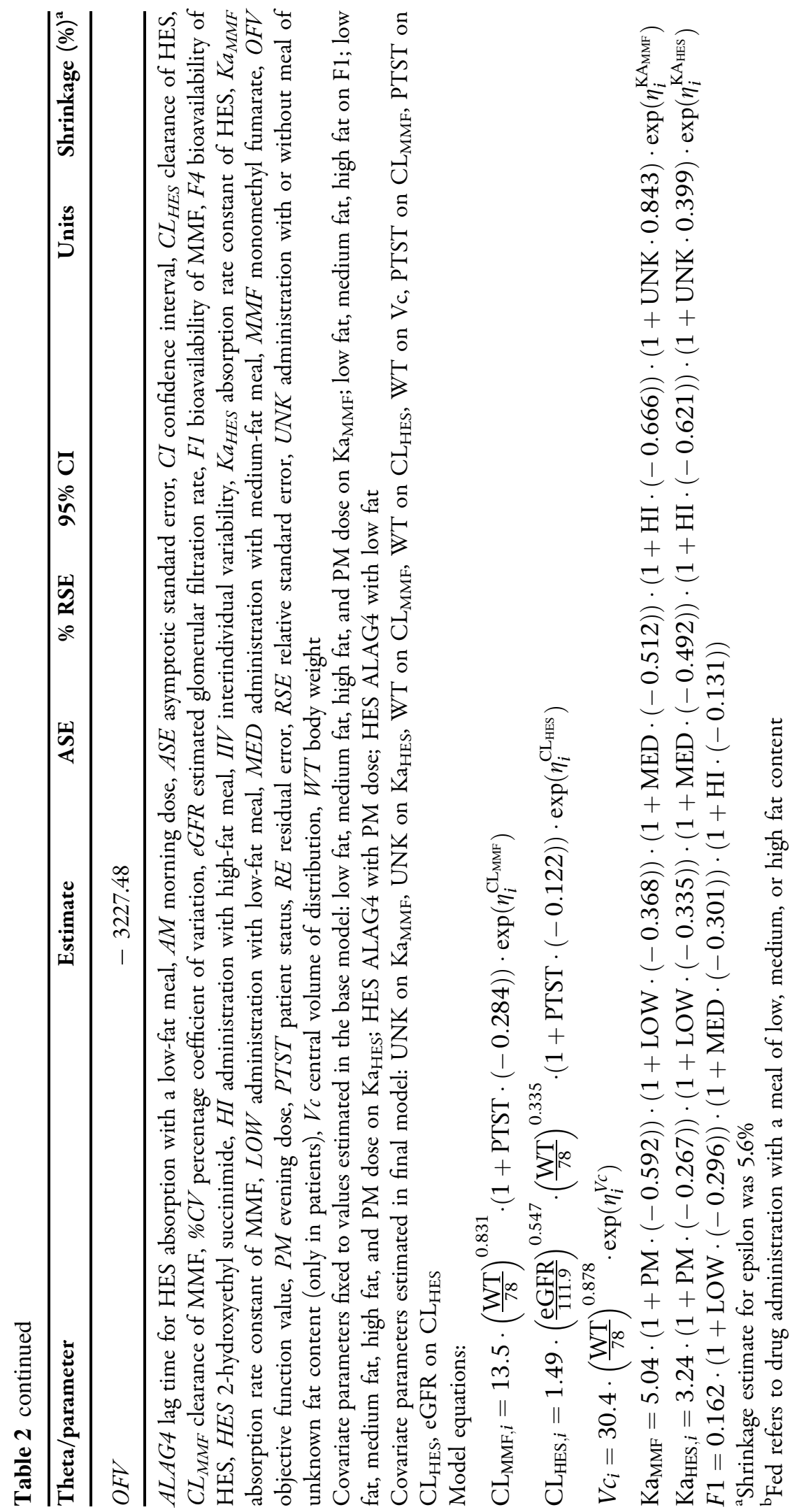


A

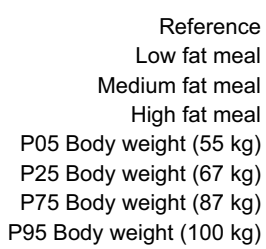

P95 Body weight $(100 \mathrm{~kg})$

0.0

Reference Low fat meal Medium fat meal High fat meal P05 Body weight $(55 \mathrm{~kg})$ P25 Body weight $(67 \mathrm{~kg})$ P75 Body weight $(87 \mathrm{~kg})$ P95 Body weight $(100 \mathrm{~kg})$

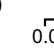

Cmax $_{0-12 h, s s}$

Covariate Effects on MMF Cmax ${ }_{0-12 h, s s}$.

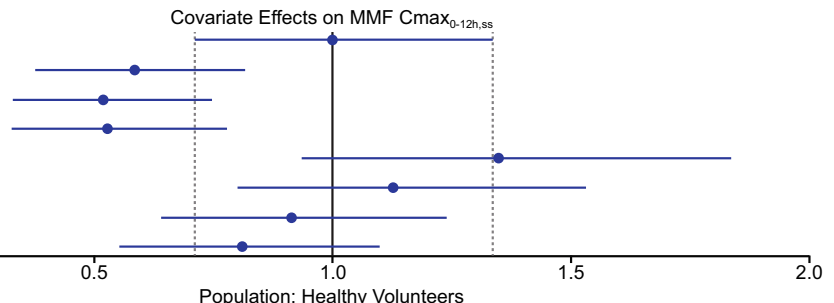

Population: Healthy Volunteers

$\mathrm{AUC}_{0-12 h, s s}$

Covariate Effects on MMF $A \cup C_{0-12 h, s s}$

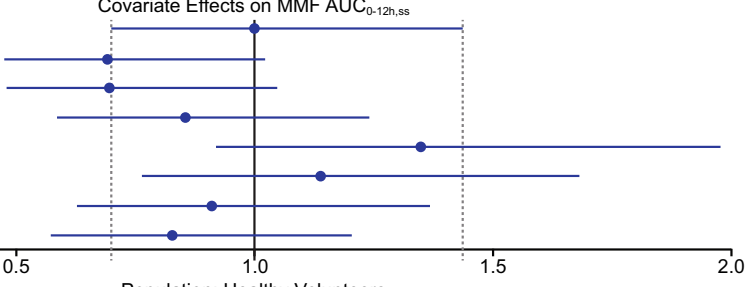

Population: Healthy Volunteers

Reference Low fat meal Medium fat meal High fat meal P05 Body weight $(55 \mathrm{~kg})$ P25 Body weight $(67 \mathrm{~kg})$

P75 Body weight $(87 \mathrm{~kg})$ P95 Body weight $(100 \mathrm{~kg})$ Normal renal function $(90-120 \mathrm{~mL} / \mathrm{min})$ Mild renal impairment $(60-89 \mathrm{~mL} / \mathrm{min})$ Moderate renal impairment $(30-59 \mathrm{~mL} / \mathrm{min})$

Severe renal impairment $(15-29 \mathrm{~mL} / \mathrm{min})$

Reference Low fat meal Medium fat meal High fat meal P05 Body weight $(55 \mathrm{~kg})$ P25 Body weight $(67 \mathrm{~kg})$ P75 Body weight $(87 \mathrm{~kg})$ P95 Body weight $(100 \mathrm{~kg})$ Normal renal function $(90-120 \mathrm{~mL} / \mathrm{min})$ Mild renal impairment $(60-89 \mathrm{~mL} / \mathrm{min})$ Moderate renal impairment $(30-59 \mathrm{~mL} / \mathrm{min})$ Severe renal impairment $(15-29 \mathrm{~mL} / \mathrm{min})$
Cmax $_{0-12 h, s s}$

Covariate Effects on HES Cmax ${ }_{0-12 h, s s}$

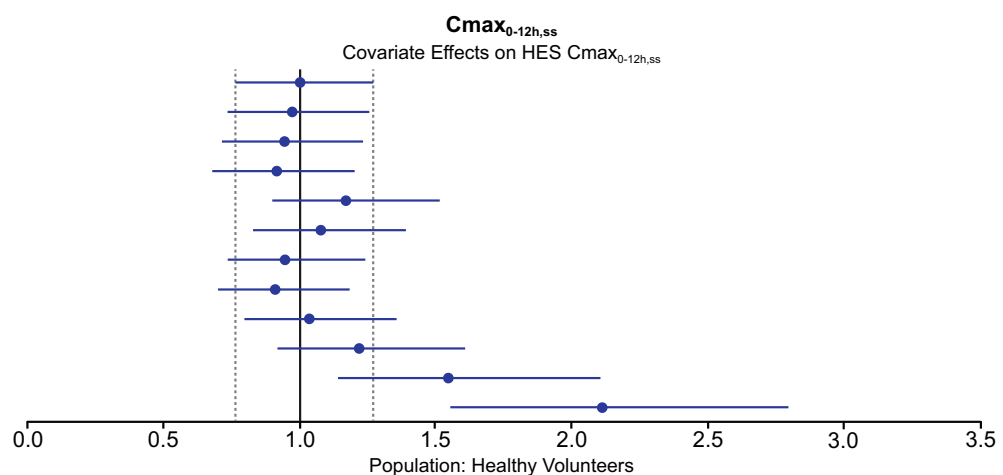

AUC $_{0-12 h, s s}$

Covariate Effects on HES AUC $\mathrm{C}_{0.12 h, s s}$

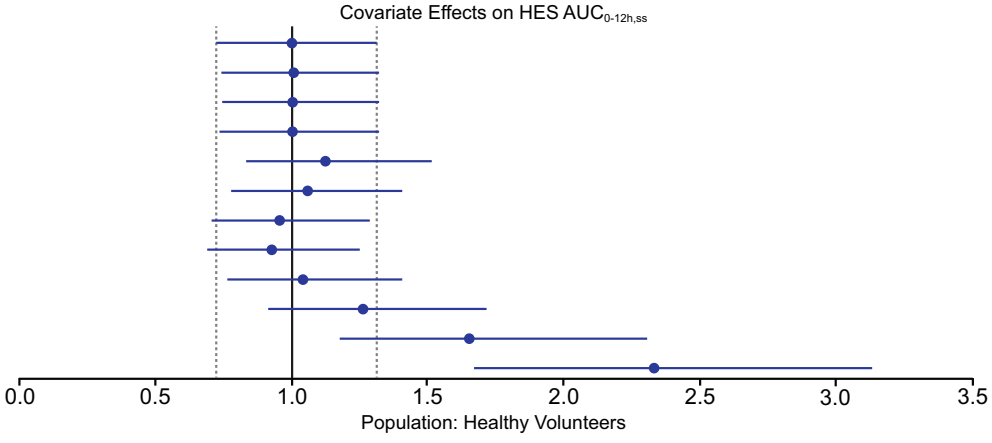


4Fig. 2 Illustration of covariate effects on steady state exposure of MMF (a) and HES (b) in healthy participants. Blue circles show the ratio of the median parameter value under the test conditions compared with the reference healthy participant with median body weight of $78 \mathrm{~kg}$ (and median eGFR of $111.9 \mathrm{~mL} / \mathrm{min}$ ), for (b), receiving DRF in a fasted state. Test conditions for body weight include the 5th, 25th, 75th, and 95th percentiles of body weight among participants in the analysis dataset. Test conditions for dietary fat include administration of DRF with a low-, medium-, and high-fat meal. Test conditions for renal function include four values of eGFR within each renal function category: normal $(\mathrm{eGFR}=120,110,100$, $90 \mathrm{~mL} / \mathrm{min}$ ); mild impairment (eGFR $=89,80,70$, $60 \mathrm{~mL} / \mathrm{min})$; moderate impairment $(59,50,40,30 \mathrm{~mL} /$ $\mathrm{min})$; and severe impairment (eGFR $=29,25,20,15 \mathrm{~mL} /$ min), summarized within each category. The blue line segments represent the corresponding 90\% prediction interval for the reference conditions. Vertical dashed lines indicate the $90 \%$ prediction interval for the reference conditions. Simulations $(N=1000)$ were performed for virtual participants (one per test condition and reference), with parameter values fixed to the final model parameter estimates and incorporating interindividual variability (i.e., individual population-predicted-derived concentration-time profiles were generated). $\mathrm{AUC}_{0-12 \mathrm{~h}, \mathrm{ss}}$ steady state area under the plasma drug concentration-time curve over the $0-12$-h dosing interval, $C_{\max 0-12 \mathrm{~h}, \mathrm{ss}}$ steady state maximum plasma drug concentration over the $0-12-\mathrm{h}$ dosing interval, DRF diroximel fumarate, eGFR estimated glomerular filtration rate, HES 2-hydroxyethyl succinimide, MMF monomethyl fumarate, P05 5th percentile, P25 25th percentile, P75 75th percentile, P95 95th percentile

performed for a virtual reference participant and virtual participants with selected test conditions using parameter estimates from the final model and incorporating interindividual variability.

Visualizations of covariate effects for healthy participants using forest plots are shown in Fig. 2. Similar plots for patients with MS are shown in Fig. S1 of the ESM.

\section{Effect of Body Weight}

Baseline body weight had a significant effect on the CL and Vc of MMF and HES in the model. Compared with a participant of median body weight $(78 \mathrm{~kg}), \mathrm{CL}_{\mathrm{MMF}}$ in participants with low
(55 kg) and high (100 kg) body weight was 25\% lower and 23\% higher, respectively, with low and high values representing 5th and 95th percentiles for body weight in the dataset, respectively. $\mathrm{CL}_{\mathrm{HES}}(11 \%$ lower and $9 \%$ higher with low and high body weight, respectively) and combined metabolite Vc $(26 \%$ lower and $24 \%$ higher with low and high body weight, respectively) were predicted to be similarly affected relative to median body weight.

Relative to participants with median body weight $(78 \mathrm{~kg})$ who received the same dose of DRF, participants with low body weight are predicted to have steady state exposure $\left(\mathrm{AUC}_{0-12 \mathrm{~h}, \mathrm{ss}}\right)$ that is $32 \%$ higher for MMF and $14 \%$ higher for HES, while participants with high body weight are predicted to have exposure that is $19 \%$ lower for MMF and $8 \%$ lower for HES (Fig. 2).

\section{Effect of Renal Function}

Renal function based on eGFR was a statistically significant predictor of $\mathrm{CL}_{\mathrm{HES}}$, consistent with urinary excretion as the primary elimination pathway for this metabolite. Changes in $\mathrm{CL}_{\mathrm{HES}}$ with renal function resulted in 1.2-fold, 1.5fold, and approximately 2-fold increases in median $C_{\text {max0-12h,ss, }}$ and 1.2-fold, 1.6-fold, and approximately 2-fold increases in median $\mathrm{AUC}_{0-12 \mathrm{~h}, \mathrm{ss}}$ for participants with mild (eGFR 60-89 mL/min), moderate (eGFR 30-59 mL/ $\mathrm{min}$ ), or severe renal impairment (eGFR $15-29 \mathrm{~mL} / \mathrm{min})$, respectively, compared with participants with normal renal function (eGFR $\geq 90 \mathrm{~mL} / \mathrm{min}$ ). The predicted values are generally consistent with the observed increase in HES exposure up to 2.5 -fold with severe renal impairment in Study A108.

\section{Effect of Food}

Factors known to influence the absorption of MMF and HES were incorporated as structural covariates in the base model and then fixed to the estimated values in development of the final model. These factors included reduced absorption rate constant with dietary fat $(37 \%, 51 \%$, and $67 \%$ lower $\mathrm{Ka}_{\mathrm{MMF}}$ and $34 \%, 49 \%$, and $62 \%$ 
A

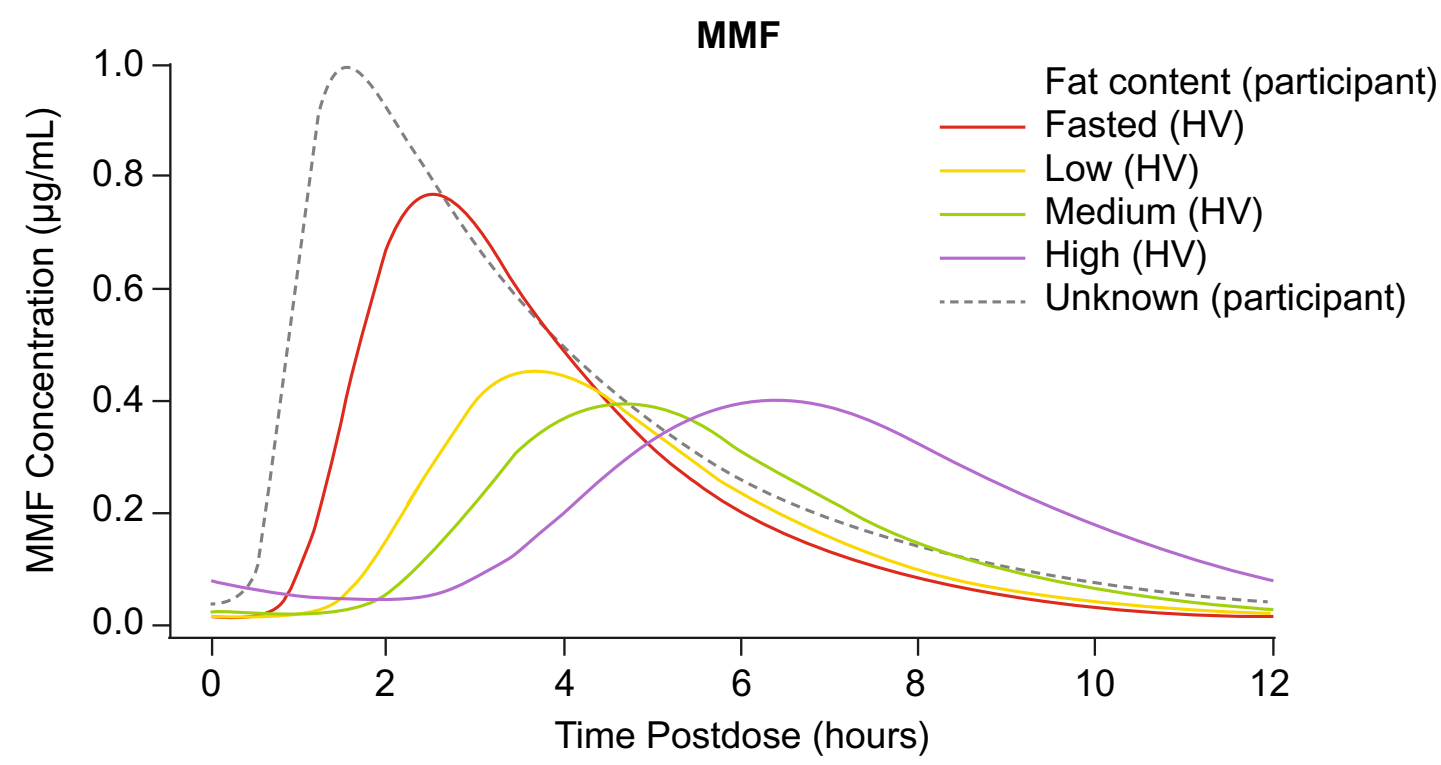

B

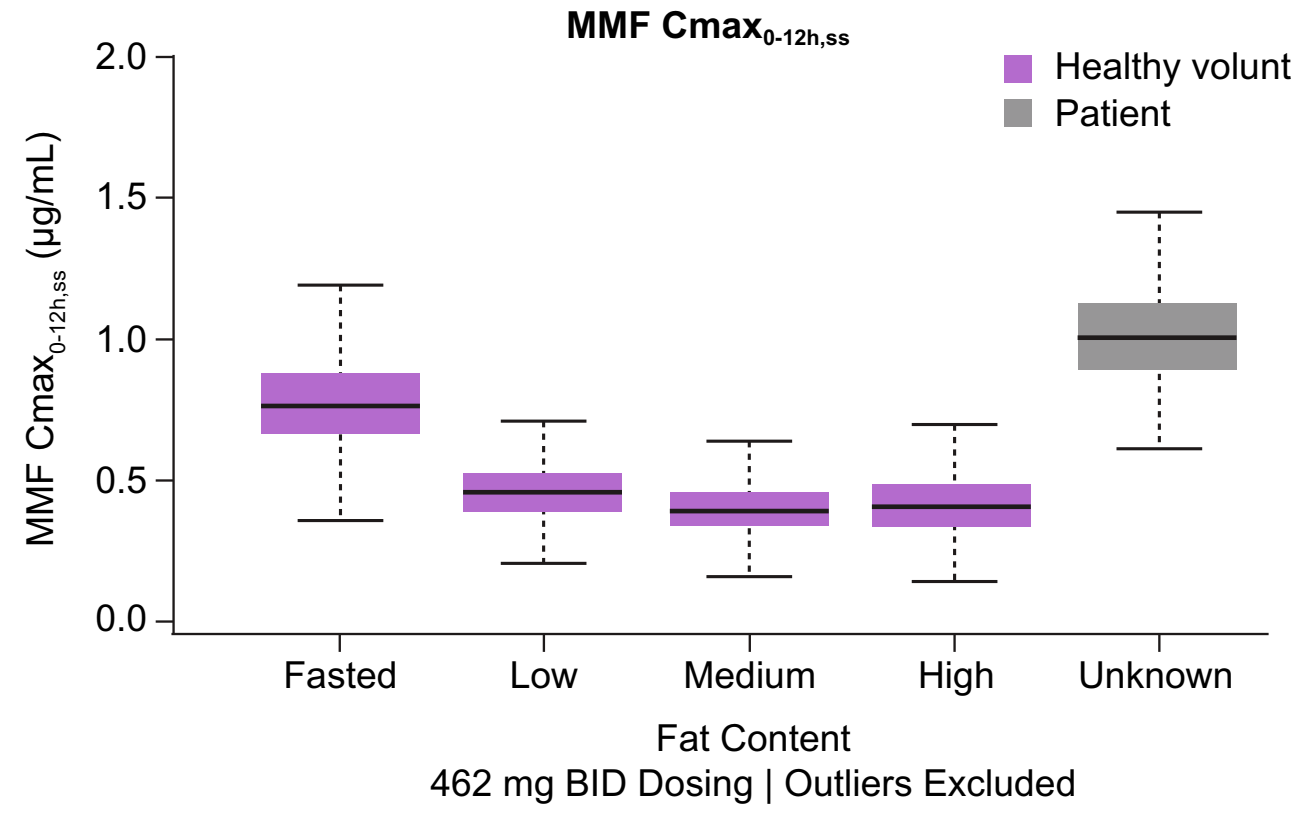

Fig. 3 Model-based simulation of MMF concentra-

$111.9 \mathrm{~mL} / \mathrm{min}$. Simulation: four virtual healthy particition-time profiles (a) and MMF $C_{\max 0-12 \mathrm{~h}, \mathrm{ss}}(\mathbf{b})$ with meal fat content in healthy participants and patients with MS following administration of DRF $462 \mathrm{mg}$ BID. Reference: in the simulations for a, participants had body weight of $78 \mathrm{~kg}$ and eGFR of $111.9 \mathrm{~mL} / \mathrm{min}$. Reference: in the simulations for $\mathbf{b}$, individual population-predicted-derived concentration-time profiles were generated for participants with body weight of $78 \mathrm{~kg}$ and eGFR of pants (one fasted and on per meal fat content category: low, medium, high) and one virtual patient (unknown meal fat content); $N=1000$ per participant. Dose: $462 \mathrm{mg}$ DRF BID for 7 days. BID twice daily, $C_{\max 0-}$ $12 \mathrm{~h}, \mathrm{~s}$, steady state maximum concentration for the $0-12$-h dosing interval following a morning dose, DRF diroximel fumarate, eGFR estimated glomerular filtration rate, HV healthy volunteer, MMF monomethyl fumarate, MS multiple sclerosis 
lower $\mathrm{Ka}_{\mathrm{HES}}$ with low-, medium-, and high-fat meals, respectively); reduced bioavailability of MMF with dietary fat (30\% lower with low/ medium fat and 13\% lower with high fat); and reduced absorption rate constants of MMF (59\% reduction) and HES (27\% reduction) with evening dosing. The lag time of $0.4 \mathrm{~h}$ estimated in the base model for HES absorption with a lowfat meal and lag time of $2 \mathrm{~h}$ for HES with evening dosing were also fixed to the base model estimates in the final model.

Model-predicted PK profiles of MMF with meal fat content are shown in Fig. 3, where delayed absorption and lower peak concentrations are observed when DRF is administered with low-, medium-, and high-fat meals compared with the fasted state. An apparent faster absorption was observed in patients with MS compared with healthy participants, in part due to DRF administration under dietary conditions that included the fasted state, although the actual meal fat content was unknown in the MS population. In EVOLVE-MS-1 and EVOLVE-MS2 , patients were instructed to take the medication with or without food, but to avoid a highfat, high-calorie meal.

\section{Effect of Morning Versus Evening Dose}

Administration of DRF in the evening resulted in delayed absorption and lower peak concentration compared with the morning dose (Fig. S2 of the ESM). The delayed absorption in the evening was more notable for HES than for MMF. However, peak concentrations were affected to a greater extent for MMF; with an evening dose, median $C_{\text {max0-12h,ss }}$ was reduced by $37 \%$ for MMF and $12 \%$ for HES relative to a morning dose.

\section{Effect of Other Covariates}

Participant age (range 18-75 years), sex, and race had no impact on MMF or HES exposure in model-based simulations. Similar findings were demonstrated for levels of total bilirubin, albumin, or AST at baseline. However, an effect was observed for participant status (healthy volunteer or patient with MS). In patients with MS,
CL was $28 \%$ and $12 \%$ lower for MMF and HES, respectively, compared with healthy participants. The absorption rate was significantly higher in patients with MS than in healthy participants (MMF, 1.8-fold; HES, 1.4-fold).

\section{DISCUSSION}

Here we report development of a population PK model for the DRF metabolites MMF and HES using pooled concentration-time data from 11 clinical studies of DRF conducted in healthy participants and patients with RRMS. DRF metabolites showed linear kinetics across dose levels studied and over time, based on population PK modeling. The population PK of MMF and HES was adequately described using a joint metabolite model that included one-compartment disposition, transit compartment absorption, and linear elimination for MMF and HES. In general, residual error estimates were higher for MMF compared with HES, which is consistent with higher variability observed for MMF concentrations. However, despite this variability, the AUC of MMF after DRF and DMF administration are remarkably stable across studies, and the variability of MMF PK is expected to have no effect on efficacy at the recommended dose of DRF or DMF $[5,15,16]$.

Covariate testing in model-based simulations revealed that the PK of MMF and HES was impacted by factors related to DRF dose administration. Previous PK analyses with DMF in healthy volunteers and patients with MS have demonstrated the impact of meal fat content on MMF exposure (AUC) and peak concentration $\left(C_{\max }\right)$, with no effect on AUC but decreased $C_{\max }$ when DMF is administered with a high-fat, high-calorie meal [4]. DRF is bioequivalent to DMF in fasted conditions [5] and a comparison of MMF PK profiles following DRF and DMF under various food conditions has been previously reported [17]. Administration of DRF with low-, medium-, and high-fat meals resulted in $12 \%, 25 \%$, and $44 \%$ reduction in MMF $C_{\max }$ without significant effect on exposure [2]. Overall, the range of MMF $C_{\max }$ values produced by DRF fell within the range of MMF $C_{\max }$ values produced by DMF with or without 
food; hence, this finding is not expected to be clinically relevant, as $C_{\max }$ values occurred within the established therapeutic range of DMF values. Administering DRF with food is not expected to have a clinically significant effect on the pharmacokinetics of MMF.

Time of dose was found to affect the PK of MMF and HES, with slower absorption rates (59\% and 27\% reduction for MMF and HES, respectively) observed with the DRF evening dose compared with the morning dose. Administration of the DRF evening dose also resulted in an estimated 2-h lag time with HES. Similar effects were observed for MMF administered as DMF [15]. It is likely that delayed absorption, which is observed with food and fumarates, had occurred with meals taken during the evening. Given that such a decrease did not affect DMF efficacy, which was also administered twice daily in the pivotal trials compared with placebo, it should be assumed that similarly, this delay in absorption and decreased absorption rate will not affect the efficacy of DRF.

Several patient characteristics, including body weight, extent of renal function, and status of patient with MS, were also shown to impact PK profiles. Model-based simulations demonstrated that baseline body weight was a significant covariate for both MMF and HES. As body weight decreases, CL and Vc also decrease, resulting in higher exposures in participants with lower body weight. PK parameters for HES seem to be less sensitive to body weight changes than parameters for MMF are. Participants with low body weight $(55 \mathrm{~kg})$ are predicted to have increased steady state exposure $\left(\mathrm{AUC}_{0-12 \mathrm{~h}, \mathrm{ss}}\right.$; $32 \%$ higher for MMF and 14\% higher for HES), while patients with high body weight $(100 \mathrm{~kg})$ are predicted to have decreased exposure (19\% lower for MMF and 8\% lower for HES), relative to the median body weight of $78 \mathrm{~kg}$ for an adult in the population analysis dataset.

Although body weight was found to impact PK parameters of MMF and HES, this impact is not expected to be clinically relevant, and no dose adjustments based on body weight for DRF are required. This conclusion is based on findings with DMF, the reference drug for DRF, which also exhibits a statistically significant body weight effect on AUC and $C_{\max }$ of MMF [18]. Clinical trials with DMF included patients of weight ranging from 34.0 to $162.3 \mathrm{~kg}$ [18]; on the basis of an analysis of covariance (ANCOVA) model, MMF AUC decreased by about $2 \%$ and MMF $C_{\max }$ decreased by about $1.4 \%$ with each $1-\mathrm{kg}$ increase in weight. Although weight had a significant effect on PK parameters for MMF in the current study, results from two pivotal phase III studies (DEFINE and CONFIRM) in patients with MS using DMF indicated that differences in patient body weight did not have an effect on the efficacy $[4,6,7]$. Therefore, no clinically relevant impact is expected for DRF, and no weight-based dose adjustment is considered to be necessary, because the therapeutic dose for DRF (462 mg, administered as $231 \mathrm{mg}$ twice daily) provides the bioequivalent MMF exposure to that achieved with the approved DMF dose of $240 \mathrm{mg}$ twice daily (equalling a daily total of $480 \mathrm{mg}$ ) [2, 4, 15]. Once daily administration was not explored as part of the DEFINE and CONFIRM studies; as the terminal half-life of MMF is approximately $1 \mathrm{~h}$ with no accumulation with multiple dosing, a once daily dose may be sub-optimal.

Baseline eGFR, a measure of renal function, had a statistically significant effect on $\mathrm{CL}_{\mathrm{HES}}$. These findings are consistent with the known renal elimination pathway of HES. Model-based simulations demonstrated greater fold increases in HES $C_{\text {max0-12h,ss }}$ and $\mathrm{AUC}_{0-12 \mathrm{~h}, \mathrm{ss}}$ with greater extent of renal impairment (mild, moderate, severe) compared with participants with normal renal function. Although there is some impact on $\mathrm{CL}_{\mathrm{HES}}$ in participants with impaired renal function, this is not considered to be clinically relevant as HES is an inactive metabolite. However, given the lack of long-term safety data in patients with moderate to severe renal impairment, DRF is currently not recommended for use in this population.

Interestingly, participant status also affected the CL and Vc of MMF and HES, with patients with MS experiencing 28\% and 12\% lower CL for MMF and HES, respectively, compared with healthy participants, suggesting higher exposure in patients with MS. It is not fully understood why MMF clearance in patients with MS appears to be lower than in healthy volunteers, 
as MMF metabolism is mediated via the tricarboxylic acid (TCA) cycle and rates of TCA-driven clearance across different diseases have not been reported. However, it is worth noting that following DRF and DMF administration, daily AUC and $C_{\max }$ estimates of MMF are nearly identical in healthy volunteers and patients with MS, and that MMF AUC and $C_{\max }$ are bioequivalent in healthy volunteers. Consequently, one may conclude that such differences in MMF PK between healthy volunteers and patients with MS may be related to differences in rates of MMF conversion, rates of MMF binding to its target, or abundance of MMF target in patients with MS. Absorption rates of MMF and HES were also significantly higher in patients with MS than in healthy participants; however, there is no clear reason behind this effect.

There was no effect of participant age, sex, or race on MMF and HES exposure. Both eGFR and sex were tested as covariates during the model development. HES PK is not affected by sex beyond its contribution to GFR estimation; MMF PK is not affected by eGFR or sex. Because MMF is metabolized through the TCA cycle, without cytochrome $\mathrm{P} 450$ involvement, hepatic impairment would not be expected to affect the PK of MMF and HES, and this is consistent with the finding from the population PK analysis that liver function parameters such as baseline total bilirubin, baseline albumin, and baseline AST were not found to be significant covariates.

There were limitations to the study. First, there was a possible confounding effect of the evening dose with fasting status, as study A102 included PK from evening doses that did not require fasting prior to dosing. Additionally, the majority of data used in the population PK model were collected in healthy volunteers rather than in patients with MS. Furthermore, the data from patients with MS that were collected reflect DRF administration with an unknown fat content since patients in the phase III studies were instructed to take DRF with or without food, which may have contributed to MMF PK variability and possibly underpredicted $C_{\max }$. Differences in dietary instructions between participant groups also limit the ability to distinguish between the contributions of disease status and meal fat content to PK parameters, since healthy volunteers received specific direction around meal fat content or fasting. The effect of disease status on PK parameters may not be separable from the effects of covariates including meal routine. The possible existence of a food-disease interaction may be a further potential influence on PK parameters, whereby the effect of food or fat content on PK acts differently in patients with MS than in healthy participants.

The influence of patient status may be, at least in part, explained by additional covariates not studied here. We also observed high residual error estimates for MMF of $90-112 \%$. Finally, it is possible that other covariates outside the scope of those studied here could have affected the assessed PK parameters.

\section{CONCLUSIONS}

A joint metabolite model adequately characterized the concentration-time data for MMF and HES following DRF administration to healthy participants and patients with MS. Meal fat content, evening dosing, participant body weight, and extent of renal impairment were all determined to impact MMF and/or HES PK profiles in final model-based simulations but not expected to be clinically significant.

\section{ACKNOWLEDGEMENTS}

Funding. This study and the journal's Rapid Service Fee was funded by Biogen.

Medical Writing Assistance. Susan Chow, PhD, from Excel Scientific Solutions, wrote sections of the first draft of the manuscript based on input from authors, and Nathaniel Hoover, from Excel Scientific Solutions, copyedited and styled the manuscript per journal requirements. Biogen provided funding for medical writing support in the development of this manuscript. 
Authorship. All named authors meet the International Committee of Medical Journal Editors (ICMJE) criteria for authorship for this article, take responsibility for the integrity of the work as a whole, and have given their approval for this version to be published.

Author Contributions. Mita Kuchimanchi, Howard Bockbrader, Lauren Quinlan, Sunny Chapel, Natasha Penner: analysis and interpretation of data, statistical analysis, drafting/critically revising manuscript. Nancy Dolphin, Daniel Epling: analysis and interpretation of data, drafting/critically revising manuscript.

Disclosures. Mita Kuchimanchi was a fulltime employee of Biogen at the time the analysis was conducted, holds stock/stock options in Biogen, and is currently an employee of GSK. Nancy Dolphin and Daniel Epling are full-time employees of A2PG and consultants for Biogen. Howard Bockbrader is a contractor for A2PG and consultant for Biogen. Lauren Quinlan was a full-time employee of A2PG at the time the analysis was conducted and is currently an employee of Critical Path Institute. Sunny Chapel is Chief Executive Officer of A2PG and a consultant for Biogen. Natasha Penner was a full-time employee of Biogen at the time the analysis was conducted, holds stock/stock options in Biogen, and is currently an employee of Zentalis Pharmaceuticals.

Compliance with Ethics Guidelines. All clinical studies included in this analysis were conducted in accordance with local and central ethics committees, the Declaration of Helsinki, and the International Conference on Harmonisation Guidelines for Good Clinical Practice. All study participants provided written informed consent.

Data Availability. The datasets generated during and/or analyzed during the current study are available from the corresponding author on reasonable request.

Open Access. This article is licensed under a Creative Commons Attribution-NonCommercial 4.0 International License, which permits any non-commercial use, sharing, adaptation, distribution and reproduction in any medium or format, as long as you give appropriate credit to the original author(s) and the source, provide a link to the Creative Commons licence, and indicate if changes were made. The images or other third party material in this article are included in the article's Creative Commons licence, unless indicated otherwise in a credit line to the material. If material is not included in the article's Creative Commons licence and your intended use is not permitted by statutory regulation or exceeds the permitted use, you will need to obtain permission directly from the copyright holder. To view a copy of this licence, visit http:// creativecommons.org/licenses/by-nc/4.0/.

\section{REFERENCES}

1. Doshi A, Chataway J. Multiple sclerosis, a treatable disease. Clin Med (Lond). 2016;16:s53-9.

2. Biogen: VUMERITY $®$ (diroximel fumarate) delayedrelease capsules, for oral use. 2021. https://www. vumerity.com/content/dam/commercial/vumerity/ pat/en_us/pdf/vumerity-prescribing-information. pdf. Accessed 18 Nov 2021.

3. Palte MJ, Wehr A, Tawa M, et al. Improving the gastrointestinal tolerability of fumaric acid esters: early findings on gastrointestinal events with diroximel fumarate in patients with relapsingremitting multiple sclerosis from the phase 3, openlabel EVOLVE-MS-1 study. Adv Ther. 2019;36: 3154-65.

4. Biogen: TECFIDERA ${ }^{\circledR}$ (dimethyl fumarate) delayedrelease capsules, for oral use. 2021. https://www. tecfidera.com/content/dam/commercial/tecfidera/ pat/en_us/pdf/full-prescribing-info.pdf. Accessed 18 Nov 2021.

5. Wehr A, Hard M, Yu M, Leigh-Pemberton R, von Moltke L. Relative bioavailability of monomethyl fumarate after administration of ALKS 8700 and dimethyl fumarate in healthy subjects. Neurology. 2018;90:P1.403.

6. Gold R, Kappos L, Arnold DL, et al. Placebo-controlled phase 3 study of oral BG-12 for relapsing multiple sclerosis. N Engl J Med. 2012;367: 1098-107. 
7. Fox RJ, Miller DH, Phillips JT, et al. Placebo-controlled phase 3 study of oral BG-12 or glatiramer in multiple sclerosis. N Engl J Med. 2012;367:1087-97.

8. Gold R, Arnold DL, Bar-Or A, et al. Safety and efficacy of delayed-release dimethyl fumarate in patients with relapsing-remitting multiple sclerosis: 9 years' follow-up of DEFINE, CONFIRM, and ENDORSE. Ther Adv Neurol Disord. 2020;13: 1756286420915005.

9. Hellwig K, Rog D, McGuigan C, et al. Pregnancy outcomes following exposure to dimethyl fumarate: interim analysis of a prospective international registry. In: 37th Congress of the European Committee for Treatment and Research in Multiple Sclerosis, Oct 13-15, 2021.

10. Phillips JT, Selmaj K, Gold R, et al. Clinical significance of gastrointestinal and flushing events in patients with multiple sclerosis treated with delayed-release dimethyl fumarate. Int J MS Care. 2015;17:236-43.

11. Fox EJ, Vasquez A, Grainger W, et al. Gastrointestinal tolerability of delayed-release dimethyl fumarate in a multicenter, open-label study of patients with relapsing forms of multiple sclerosis (MANAGE). Int J MS Care. 2016;18:9-18.

12. Naismith RT, Wundes A, Ziemssen T, et al. Diroximel fumarate demonstrates an improved gastrointestinal tolerability profile compared with dimethyl fumarate in patients with relapsing-remitting multiple sclerosis: results from the randomized, doubleblind, phase III EVOLVE-MS-2 study. CNS Drugs. 2020;34:185-96.

13. Naismith R, Wolinsky J, Wundes A, et al. Diroximel fumarate (DRF) in patients with relapsing-remitting multiple sclerosis: interim safety and efficacy results from the phase 3 EVOLVE-MS-1 study. Mult Scler J. 2020;26:1729-39.

14. Bertrand J, Laffont CM, Mentre F, Chenel M, Comets E. Development of a complex parentmetabolite joint population pharmacokinetic model. AAPS J. 2011;13:390-404.

15. Biogen: Data on file. 2020. Accessed 12 Dec 2020.

16. Wu F, Gold R, Freedman M, et al. Efficacy outcomes for diroximel fumarate compared with dimethyl fumarate: a propensity score matched analysis of EVOLVE-MS-1 and DEFINE/CONFIRM. In: 5th Congress of the European Academy of Neurology, 2019, Oslo, Norway.

17. Penner N, von Moltke L, Hard M. Effect of food on the pharmacokinetics of monomethyl fumarate after administration of diroximel fumarate. San Antonio: American Academy of Pharmaceutical Scientists (AAPS) PharmSci 360; Nov 3-6, 2019.

18. Biogen: Data on file. 2021. Accessed 18 Nov 2021. 